\title{
Organic Aerosols in Anoxic and Oxic Atmospheres of Earth-like Exoplanets: VUV-MIR Spectroscopy of CHON Tholins
}

\author{
Lisseth Gavilan $^{1}$ (D), Nathalie Carrasco ${ }^{1}$ (1), Søren Vrønning Hoffmann ${ }^{2}$, Nykola C. Jones ${ }^{2}$, and Nigel J. Mason ${ }^{3}$ \\ ${ }^{1}$ LATMOS, Université Versailles St. Quentin, UPMC Université Paris 06, CNRS, 11 Blvd. d'Alembert, F-78280 Guyancourt, France; lisseth.gavilan@gmail.com \\ ${ }^{2}$ ISA, Department of Physics and Astronomy, Aarhus University, Ny Munkegade 120, DK-8000 Aarhus C, Denmark \\ ${ }^{3}$ Department of Physical Sciences, The Open University, Milton Keynes MK7 6AA, UK \\ Received 2018 February 5; revised 2018 May 4; accepted 2018 May 28; published 2018 July 11
}

\begin{abstract}
Organic hazes can drastically impact the characterization and detection of exoplanet atmospheres. We explore the effects of the transition from an anoxic to oxic atmosphere on the molecular structure of organic aerosols, by producing aerosols in $\mathrm{N}_{2}: \mathrm{CO}_{2}$ : $\mathrm{CH}_{4}$-rich plasmas with increasing $\mathrm{CO}_{2} / \mathrm{CH}_{4}$ gas ratios. We performed a spectroscopic study on the resulting aerosol analogs, CHON-rich tholins, from the vacuum-ultraviolet (VUV) to the mid-infrared (MIR), i.e., the $0.13-10 \mu \mathrm{m}$ range. VUV spectroscopy revealed the presence of $\pi-\pi^{*}$ and $n-\pi^{*}$ transitions in the $200-500 \mathrm{~nm}$ range in all samples. These are attributed to electronic transitions in amine groups. As the $\mathrm{CO}_{2} / \mathrm{CH}_{4}$ ratio increases, new bands emerge that can be attributed to electronic transitions in hydroxyl and carboxyl functional groups. MIR spectroscopy showed that the molecular structure of oxidized aerosols is dominated by the $\mathrm{CO}_{2} / \mathrm{CH}_{4}$ ratio. Band deconvolution revealed the oxygenation of the organic matrix via the formation of oxygenated functional groups, including amide, hydroxyl, and carbonyl groups. For the most oxidized aerosols, absorption is greatest in the $0.13-0.3 \mu \mathrm{m}$ and $6-10 \mu \mathrm{m}$ regions. Some of the oxidized samples were further irradiated with VUV photons, resulting in the formation of $\pi-\pi^{*}$ bands in the 200-250 nm region and a decrease in the band intensities below $200 \mathrm{~nm}$, attributed to amine and nitrile losses. We discuss how oxidized aerosols could provide a detectable (bio-)signature in exoplanet atmospheres.
\end{abstract}

Key words: planets and satellites: - fundamental parameters - methods: laboratory: solid state - planets and satellites: atmospheres - techniques: spectroscopic

\section{Introduction}

Photochemical aerosols are present across the atmospheres of several bodies in the solar system. These can be observed as hazes from the stratosphere to the ionosphere. As such, and depending on their optical properties, morphology and size distribution, they could heavily impact the radiative transfer of solar radiation and planetary surface temperature. One of the most notable examples of an atmosphere dominated by photochemical aerosols is Titan. Its quasi-reducing atmospheric chemistry is the result of photon and energetic particles (protons, cosmic rays) impact on $\mathrm{N}_{2}$ and $\mathrm{CH}_{4}$, an efficient gas mixture for the generation of organic photochemical aerosols (Wilson \& Atreya 2004; Lavvas et al. 2013).

On Earth, organic aerosols are presently one of the largest drivers of radiative forcing, affecting the Earth's thermal balance and providing a mechanism for climate change (Jacobson et al. 2000). In the Archean Earth ( 4-2.5 Gyr ago), the presence of photochemical aerosols could have influenced early planetary habitability via radiative shielding and surface cooling (Pavlov et al. 2001). Unlike Titan's anoxic atmosphere, $\mathrm{CO}_{2}$ is expected to have been present in the atmosphere of Earth from its early stages following the emergence of life (Kasting 1993; von Paris et al. 2008; Feulner 2012). Atmospheric oxidation therefore probably impacted the formation and optical properties of early organic hazes (Haqq-Misra et al. 2008; Wolf \& Toon 2010).

Aerosols are likely to be ubiquitous in the atmospheres of extrasolar planets (Marley et al. 2013) and their composition can be as widely varying as that of the exoplanetary atmospheres in which they are formed (Lavvas \& Koskinen 2017). Methane was detected in the atmosphere of exoplanet HD 189733b
(Swain et al. 2008) with $\mathrm{CO}, \mathrm{CO}_{2}$, and $\mathrm{H}_{2} \mathrm{O}$ (Swain et al. 2009b). Swain et al. (2009a) detected $\mathrm{H}_{2} \mathrm{O}, \mathrm{CH}_{4}$, and $\mathrm{CO}_{2}$ in the near-infrared spectrum of exoplanet $\mathrm{HD} 209458 \mathrm{~b} . \mathrm{CO}_{2}$ photodissociation is relevant to the radiative transfer in exoplanet atmospheres (Venot et al. 2018). Its presence could also affect the photochemical production of organic hazes in Earth-like exoplanets as shown by models (Arney et al. 2017) and experiments (Trainer et al. 2006; Fleury et al. 2017; Gavilan et al. 2017).

While the optical properties of organic aerosols are needed to refine models of the Archean Earth and exoplanet atmospheres, their molecular properties are necessary to understand the spectral variations as a function of the degree of oxidation. The aim of this work is to experimentally simulate the composition of increasingly oxidizing atmospheres to understand the impact of $\mathrm{CO}_{2}$ in the optical properties of the generated organic aerosols covering a wide spectral range, from the vacuumultraviolet to the mid-infrared. This study is a continuation of our first study on the UV-visible complex refractive indices of oxidized organic aerosols (Gavilan et al. 2017) but here we focus our study on the molecular, electronic, and absorption properties of these aerosols.

\section{Experiments}

Tholins were produced using the PAMPRE ${ }^{4}$ experimental facility, a low-pressure (0.95 mbar) radiofrequency (RF) plasma reactor (Szopa et al. 2006). This apparatus allows the simulation of the complex organic chemistry initiated by energetic particles in planetary ionospheres. The $13.56 \mathrm{MHz}$

PAMPRE: Production d'Aérosols en Microgravité par Plasmas REactifs. 
Table 1

Synthesis Parameters for Tholins Prepared at Increasing $\mathrm{CO}_{2} / \mathrm{CH}_{4}$ Ratios, Thickness, and Tauc Gap Energies

\begin{tabular}{lcccc}
\hline \hline $\begin{array}{l}\text { Gas mixture } \\
\mathrm{N}_{2}: \mathrm{CO}_{2}: \mathrm{CH}_{4}\end{array}$ & $\mathrm{CO}_{2} / \mathrm{CH}_{4}$ & $\begin{array}{c}\text { Deposit } \\
(\mathrm{s})\end{array}$ & $\begin{array}{c}\text { Thickness } \\
(\mathrm{nm})\end{array}$ & $\begin{array}{c}E_{g} \\
(\mathrm{eV})\end{array}$ \\
\hline $95: 0: 5$ & 0 & 3 & $36 \pm 7$ & $2.35 \pm 0.20$ \\
$95: 2.5: 2.5$ & 1 & 15 & $79 \pm 16$ & $2.54 \pm 0.40$ \\
$90: 5: 5$ & 1 & 20 & $79 \pm 16$ & $2.49 \pm 0.40$ \\
$95: 3: 2$ & 1.5 & 30 & $195 \pm 15$ & $3.06 \pm 0.40$ \\
$90: 6: 4$ & 1.5 & 60 & $74 \pm 40$ & $2.66 \pm 0.40$ \\
$95: 4: 1$ & 4 & 600 & $43 \pm 21$ & $3.20 \pm 0.20$ \\
$90: 8: 2$ & 4 & 480 & $33 \pm 16$ & $3.23 \pm 0.20$ \\
\hline
\end{tabular}

RF source, set to $30 \mathrm{~W}$, generates a capacitively coupled plasma fed by a gas mixture at 0.95 mbar. The aerosol analogs presented in this study were prepared in a cold plasma where neutrals have a temperature of $\sim 340 \mathrm{~K}$. However, the most observed exoplanets to date are hot giants $(\sim 800-3000 \mathrm{~K})$ and are particularly conducive to atmospheric observations (Madhusudhan et al. 2016). Future laboratory simulations under different temperatures and pressures should explore how these parameters could further impact the formation, composition, and optical properties of aerosols. This paper is a first step in the investigation of the molecular properties of oxidized organic aerosols.

Initially, a gas mixture was chosen to simulate the reducing composition of Titan's atmosphere, i.e., $\mathrm{N}_{2}: \mathrm{CH}_{4}$ with a 95:5 ratio (e.g., Mahjoub et al. 2012; Sciamma-O'Brien et al. 2012). We then simulated increasingly oxidizing atmospheres by adding $\mathrm{CO}_{2}$ to the $\mathrm{N}_{2}-\mathrm{CH}_{4}$ gas mixture. The resulting tholins, solids rich in $\mathrm{C}, \mathrm{H}, \mathrm{O}$, and $\mathrm{N}$ (CHON-rich), are produced on substrates placed on the bottom electrode of the capacitor producing the plasma. One set of samples was prepared with a gas mixture, keeping the $\mathrm{N}_{2}$ partial pressure at $90 \%$, while a second set of samples was prepared keeping the $\mathrm{N}_{2}$ partial pressure at $95 \%$. For both series, gas mixtures with $\mathrm{CO}_{2}: \mathrm{CH}_{4}$ ratios equal to $0,1,1.5$, and 4 were used. We can translate the $\mathrm{CO}_{2}: \mathrm{CH}_{4}$ ratios into $\mathrm{C} / \mathrm{O}$ ratios, used to characterize exoplanet atmospheres in terms of their carbon content (e.g., Venot et al. 2015),

$$
\frac{\mathrm{C}}{\mathrm{O}}=\frac{1 \times N_{\mathrm{CO}_{2}}+1 \times N_{\mathrm{CH}_{4}}}{2 \times N_{\mathrm{CO}_{2}}}=0.5+\frac{0.5}{\mathrm{CO}_{2}: \mathrm{CH}_{4}} .
$$

Using this conversion, we find $\mathrm{C} / \mathrm{O}$ ratios equal to $\infty, 1,0.83$, and 0.68. According to Venot et al. (2015), this $\mathrm{C} / \mathrm{O}$ range includes $\mathrm{C}$-rich $(\mathrm{C} / \mathrm{O}=\infty$ and 1$)$ and near-solar $(\mathrm{C} / \mathrm{O}=0.68)$ type atmospheres.

The gas mixtures and deposition times of tholins are shown in Table 1. Although the deposition timescales differ by a factor of 100 between experiments, we have striven to obtain a film thickness leading to sufficient $\mathrm{S} / \mathrm{N}$ in transmission over the VUV-MIR range. Additional effects like sputtering and electron impact from longer exposures to the plasma could further affect the properties of such films, and this will be the object of further studies. During deposition, bare silicon $(10 \times 10 \mathrm{~mm})$ and $\mathrm{MgF}_{2}$ windows (20 mm diameter) were placed on the bottom electrode within the PAMPRE chamber. The thickness of the deposited films is estimated from ellipsometry on the Si substrates, and the method is described in Gavilan et al. (2017). In this paper, we present the result of transmission measurements performed on the films deposited on $\mathrm{MgF}_{2}$ windows. Synchrotron and benchtop spectrophotometers were used to measure the transmission spectra of these films in the vacuum-ultraviolet (VUV; 130-250 nm), ultraviolet-visible (UV-Vis; 210-1000 nm), near-infrared (NIR; $1-3 \mu \mathrm{m}$ ), and mid-infrared (MIR; $1.43-10 \mu \mathrm{m}$ ) spectral ranges. These are described in more detail below.

Although the film thickness can vary from one substrate to the other during plasma deposition, we estimate that this variation does not exceed $30 \%$ of the thickness measured on the films deposited on Si. The estimated thickness of each film is reported in Table 1. According to Arney et al. (2017), the production of organic hazes decreases when $\mathrm{CO}_{2}: \mathrm{CH}_{4}>10$. In our study, the aerosol production rate is drastically quenched at a $\mathrm{CO}_{2}: \mathrm{CH}_{4}$ ratio of 4 .

VUV transmission spectra were measured on the AU-UV beam line at the ASTRID $2^{5}$ synchrotron at Aarhus University, Denmark (Palmer et al. 2015). Thin films deposited on $\mathrm{MgF}_{2}$ windows were placed in the lollipop sample holder, able to mount up to four samples. UV-Vis transmission spectra were measured with a bench-top UV-Vis spectrometer (Thermo Scientific Evolution 300). The overlapping spectral range was used to combine the VUV and UV-Vis data sets. One sample, the fully reduced tholin, had previously been measured in the APEX $^{6}$ chamber of the DISCO ${ }^{7}$ beam line of the SOLEIL $^{8}$ synchrotron. We confirmed that VUV spectra are reproducible between beam lines at both facilities.

Near-infrared transmission spectra were measured at the Planetary Emissivity Laboratory (DLR) using a vacuum Fourier transform infrared (FTIR) spectrometer (Bruker Vertex $80 \mathrm{~V}$ ) with a liquid nitrogen cooled $\mathrm{HgCdTe}$ (mercury cadmium telluride, MCT) detector at $1 \mathrm{~cm}^{-1}$ spectral resolution. A Bruker A480 parallel beam accessory was mounted in front of the sample holder within the Vertex $80 \mathrm{~V}$ to reduce artifacts due to beam reflections on the sample. This accessory helps collimate the beam when measuring highly refractive/reflective samples in transmission mode.

Mid-infrared transmission spectra were measured with a vacuum FTIR spectrometer (Bruker Vertex 80V) available at ASTRID2. The reduced tholin mid-infrared spectrum was measured on the iN10 infrared microscope available at SOLEIL. These were recorded at $4 \mathrm{~cm}^{-1}$ spectral resolution and were corrected with a polynomial baseline to subtract the continuum absorption.

\section{VUV and MIR Spectroscopy}

Our spectroscopic analysis involves deconvolution of the main electronic bands for the VUV-UV spectra and of the main vibrational modes in the MIR spectra. This is done via the Levenberg-Marquardt nonlinear least-squares minimization method using the IDL MPFIT routine (Markwardt 2009). We have chosen Gaussian band shapes, suitable for characterizing molecular transitions in solids. In fitting each band, the peak position and intensity are left as free parameters, while the width is varied up to $10 \%$ between spectra. Band positions and widths may shift depending on the bonding environment for each sample.

\footnotetext{
5 ASTRID: Aarhus STorage RIng in Denmark, http://www.isa.au.dk.

6 APEX: Atmospheric Pressure Experiment.

7 DISCO: Dichroism, Imaging and SpectrosCOpy.

8 SOLEIL: Source Optimisée de Lumière d'Énergie Intermédiaire du LURE.
} 


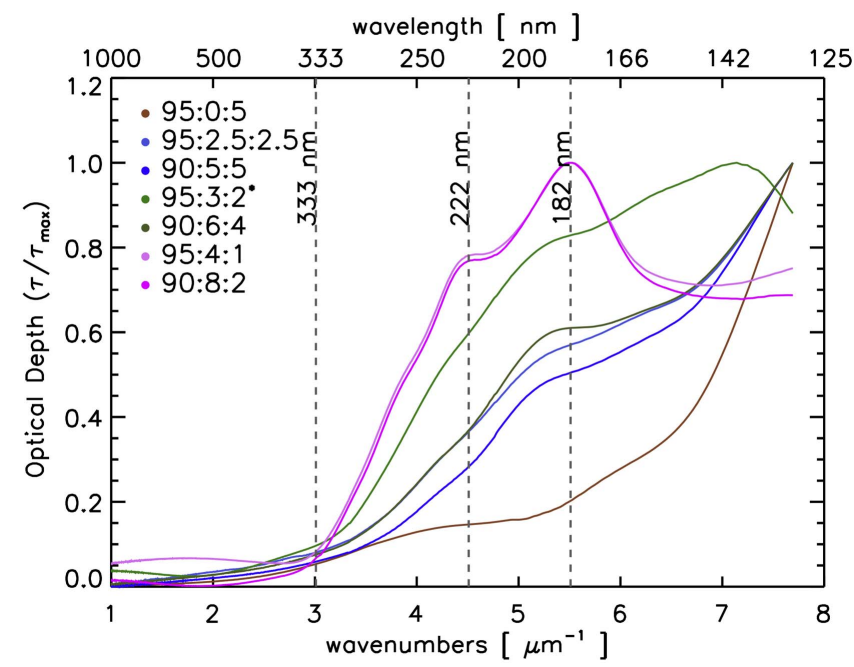

Figure 1. Vis-VUV spectra of tholins (normalized to the maximum optical depth in this range), prepared in plasmas of increasing $\mathrm{CO}_{2} / \mathrm{CH}_{4}$ ratios. Due to the large thickness $(d \sim 195 \mathrm{~nm})$, the VUV spectrum of the 95:3:2 tholin (green curve) is saturated for wavenumbers larger than $6 \mu \mathrm{m}^{-1}$.

\subsection{VUV-Visible Spectroscopy}

The VUV-visible spectra of the oxidized tholins have been normalized to the maximum optical depth in the VUV. These are shown in Figure 1. We notice that increasing the $\mathrm{CO}_{2} / \mathrm{CH}_{4}$ ratio has a direct effect on the electronic absorption bands of these tholins. This evolution is best represented by the appearance of a narrow but intense peak at $\sim 180 \mathrm{~nm}$ and a decrease in the intensity of the far-UV (FUV) band. Varying the $\mathrm{N}_{2}$ partial pressure from $90 \%$ to $95 \%$ has a negligible effect on the VUV spectra of tholins prepared with similar $\mathrm{CO}_{2} / \mathrm{CH}_{4}$ ratios.

We used the visible-near-UV spectral range $(250-1000 \mathrm{~nm})$ to determine the optical band gap of these materials, also known as the Tauc gap (Tauc et al. 1966). The gap values were obtained by extrapolating a regression from the linear region in a $(\alpha \times E)^{1 / 2}$ versus $E$ plot, where $\alpha$ is the absorption coefficient and $E$ is in $\mathrm{eV}$. The Tauc extrapolation is found using the linear region between the visible and near-ultraviolet range, i.e., 3.8-04.5 eV (see Figure 2). This region avoids the exponential absorption tail appearing in the visible energy range (1.2-3.8 eV), characteristic of disorder in amorphous materials, known as the Urbach tail (Urbach 1953; Jones 2012). We varied the endpoints of this range to determine the average systematic error in the gap determination. The gap values are listed in Table 1. We note that, as the $\mathrm{CO}_{2} / \mathrm{CH}_{4}$ ratio increases, so does the gap value, i.e., $E_{g}$ increases from 2.35 to $3.20 \mathrm{eV}$. These gap values are consistent (within the error bars) with those found via the ellipsometry on similar films deposited on Si substrates (Gavilan et al. 2017), validating the coherence of both methods.

As seen in Figure 1, the VUV-visible spectral profile for samples prepared with the same $\mathrm{CO}_{2} / \mathrm{CH}_{4}$ ratio are similar, e.g., 95:4:1 and 90:8:2 both with a ratio of 4 and 95:2.5:2.5 and 90:5:5 both with a ratio of 1 . Because the spectra of the 95:3:2 sample is optically saturated in the VUV, we chose the $90 \% \mathrm{~N}_{2}$ series to deconvolve the VUV-vis spectra for increasing $\mathrm{CO}_{2} / \mathrm{CH}_{4}$ ratios $=0,1,1.5,4$. The resulting deconvolution is shown in Figure 3.

VUV-visible spectra reveal transitions of electrons between the molecular orbitals of organic materials. Identification of possible carriers of the electronic bands in our tholins is done

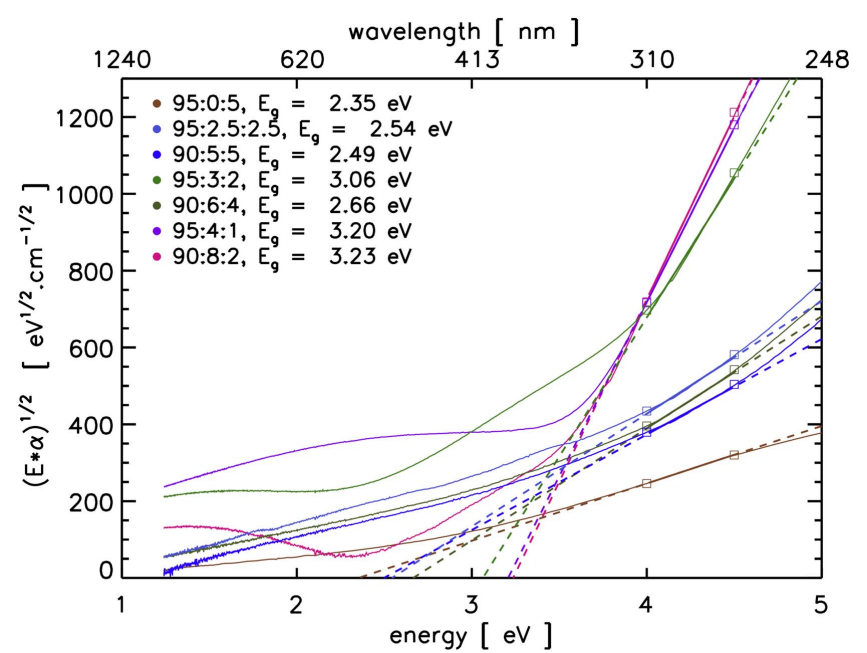

Figure 2. Tauc optical gap determination from the visible to near-ultraviolet spectra for tholins prepared in plasmas with increasing $\mathrm{CO}_{2} / \mathrm{CH}_{4}$ ratios in $\mathrm{N}_{2}: \mathrm{CO}_{2}: \mathrm{CH}_{4}$ mixtures.

by comparison to UV-Vis gas phase spectra of common chromophores present in organic molecules (e.g., Hornback 2005; Workman 2016). These are listed in Table 2. The major electronic transitions in organic compounds are $\sigma \rightarrow \sigma^{*}$, $\pi \rightarrow \pi^{*}, n \rightarrow \sigma^{*}$, and $n \rightarrow \pi^{*}$, and associated bands. Generally $\pi$-electronic transitions are found at $1.6<\lambda^{-1}<5 \mu \mathrm{m}^{-1}$ $(200<\lambda<600 \mathrm{~nm})$, while $\sigma$-electronic transitions are found at higher energies, $\lambda^{-1}>3.8 \mu \mathrm{m}^{-1}(\lambda<260 \mathrm{~nm})$ (Gadallah et al. 2011; Gavilan et al. 2016).

For our sample spectra, each VUV-Vis spectrum can be deconvolved into three main bands. However, as the oxidation degree increases, band and peak positions are modified. For the tholin with no $\mathrm{CO}_{2}$, two bands, which we call G1 and G2, are attributed to transitions in amine functional groups. G1 and G2 are assigned to electronic transitions from the lone-pair electrons on nitrogen to nitrogen atomic-like orbitals (HubinFranskin et al. 2002; Duarte et al. 2013). The largest band, called $\mathrm{G} 3$, found in the far-UV, is generally attributed to $\sigma-\sigma^{*}$ and $n-\sigma^{*}$ type transitions and its peak position is found at $8<\lambda^{-1}<8.5 \mu \mathrm{m}^{-1}(118-125 \mathrm{~nm})$.

The VUV spectra evolve as the oxidation degree increases. The spectra for samples with $\mathrm{CO}_{2} / \mathrm{CH}_{4}=1$ and 1.5 are remarkably similar. The smaller UV bands have shifted, which signals the molecular evolution of the solid. G4 is then attributed to low energy $\pi-\pi^{*}$ and $n-\pi^{*}$ transitions arising from structural disorder (Gadallah et al. 2011). G5 is a new VUV band centered at $\lambda^{-1} \sim 5.4 \mu \mathrm{m}^{-1}(185 \mathrm{~nm})$, whose intensity increases with increasing oxidation degree, and is attributed to electronic transitions in hydroxyl groups due to the promotion of an electron from the highest filled molecular orbital to an antibonding orbital $\left(\sigma_{\mathrm{OH}}^{*}\right)$ (Duarte et al. 2013). This band usually appears at $5<\lambda^{-1}<6.1 \mu \mathrm{m}^{-1}(165-200 \mathrm{~nm})$ (Xu et al. 1999).

Finally, for the most oxidized sample, with $\mathrm{CO}_{2} / \mathrm{CH}_{4}=4$, the G5 band is most prominent as a consequence of the larger number of oxygenated bonds. G6 is a new band appearing at $\lambda^{-1} \sim 4.2 \mu \mathrm{m}^{-1}(240 \mathrm{~nm})$ and is assigned to the $n_{\mathrm{O}} \rightarrow \pi_{\mathrm{CO}}^{*}$ transitions from the lone-pair orbital on the carbonyl oxygen to the antibonding $\pi_{\mathrm{CO}}$ valence orbital (Barnes \& Simpson 1963; Ari \& Güven 2000) or to the valence shell excitations of hydroxyl groups (Duarte et al. 2013). For this sample, the 


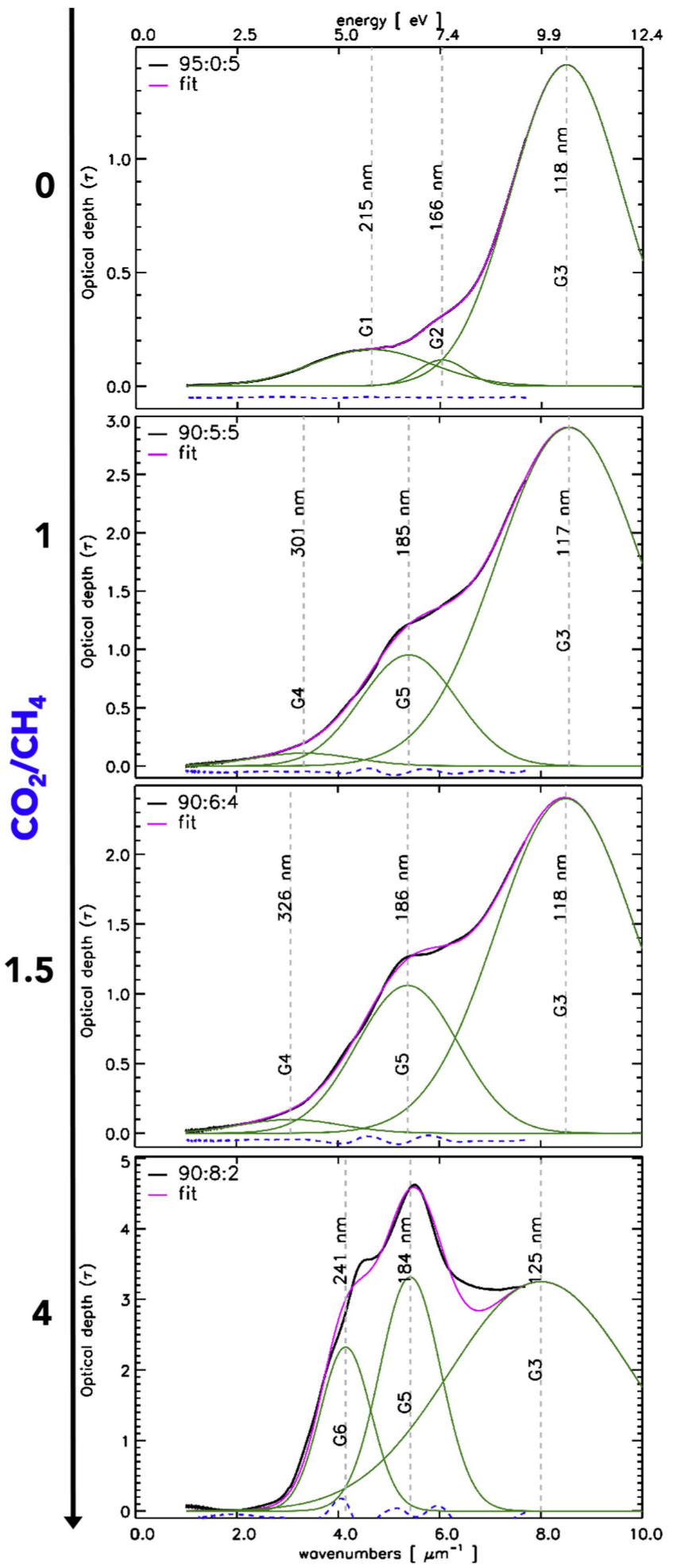

Figure 3. Spectral deconvolution of the Vis-VUV of increasingly oxidized organic tholins prepared in plasmas of increasing $\mathrm{CO}_{2} / \mathrm{CH}_{4}$ ratios.

position of G3 is shifted to lower energies and its relative intensity decreases, indicating a loss of $\sigma$-bonds.

\subsection{Mid-infrared Spectroscopy}

The mid-infrared spectra of our samples were split into three spectral ranges corresponding to the most intense vibrational
Table 2

VUV Electronic Band Assignments

\begin{tabular}{lcccl}
\hline \hline Label & $\begin{array}{c}\text { Peak Pos. } \\
\mathrm{nm} / \mathrm{eV}\end{array}$ & $\begin{array}{c}\text { Literature } \\
\text { Position }(\mathrm{eV})\end{array}$ & $\begin{array}{c}\text { Electronic } \\
\text { Transition }\end{array}$ & $\begin{array}{l}\text { Functional } \\
\text { Group }\end{array}$ \\
\hline $\mathrm{G} 1$ & $215 / 5.77$ & $5.70,^{\mathrm{a}} 5.77^{\mathrm{b}}$ & $n_{\mathrm{N}} \rightarrow 3 \mathrm{sa}$ & Amine \\
$\mathrm{G} 2$ & $166 / 7.47$ & $7.14,^{\mathrm{b}} 7.93^{\mathrm{c}}$ & $n_{\mathrm{N}} \rightarrow 3 p a$ & Amine \\
$\mathrm{G} 3$ & $118 / 10.51$ & $10.16^{\mathrm{d}}-12^{\mathrm{e}}$ & $\sigma-\sigma^{*}, n-\sigma^{*}$ & Methyl \\
G4 & $300 / 4.13$ & $3.50^{\mathrm{f}}-4.75^{\mathrm{g}}$ & $\pi-\pi^{*}, n-\pi^{*}$ & Carbonyl \\
G5 & $185 / 6.7$ & $6.2-7.5^{\mathrm{h}}$ & $n_{\mathrm{O}} \rightarrow \sigma^{*}$ & Hydroxyl \\
G6 & $241 / 5.15$ & $5.80^{\mathrm{i}}$ & $n_{\mathrm{O}} \rightarrow \pi_{\mathrm{CO}}^{*}$ & Carboxyl \\
\hline
\end{tabular}

Notes.

${ }^{\text {a }}$ Hubin-Franskin et al. (2002).

b Tannenbaum et al. (1953).

c Harshbarger (1971).

d Workman (2016).

e Gavilan et al. (2016).

${ }^{\mathrm{f}}$ Hornback (2005).

g Gadallah et al. (2011); Robertson \& O'Reilly (1987).

${ }^{\text {h }}$ Xu et al. (1999).

${ }^{\mathrm{i}}$ Barnes \& Simpson (1963); Ari \& Güven (2000).

Table 3

Mid-infrared Band Assignments for Tholins Prepared at Increasing $\mathrm{CO}_{2} / \mathrm{CH}_{4}$ Ratios

\begin{tabular}{|c|c|c|}
\hline $\begin{array}{l}\text { Peak } \\
\left(\mathrm{cm}^{-1}\right)\end{array}$ & $\begin{array}{l}\text { Range } \\
\left(\mathrm{cm}^{-1}\right)\end{array}$ & Band Assignments ${ }^{\mathrm{a}, \mathrm{b}}$ \\
\hline 3320 & $3500-3100$ & $\mathrm{NH} / \mathrm{NH}_{2}$ stretch (Amine I) \\
\hline 3200 & $\cdots$ & NH stretch (Amine I) \\
\hline 3175 & $\cdots$ & NH stretch (Amide I) \\
\hline 2960 & $3000-2800$ & $\mathrm{CH}$ stretch (ali. $\mathrm{CH}_{2} / \mathrm{CH}_{3}$, arom. $\left.=\mathrm{CH}\right)$ \\
\hline 2846 & $2900-2800$ & aldehyde $\mathrm{CH}$ stretch $(\mathrm{H}-(\mathrm{R}) \mathrm{C}=\mathrm{O})$ \\
\hline 2240 & $2260-2230$ & $\mathrm{R}-\mathrm{C} \equiv \mathrm{N}$, nitrile stretch \\
\hline 2180 & $2230-2150$ & $\mathrm{C}=\mathrm{C}-\mathrm{CN}$ conjugated nitrile stretch $^{\mathrm{c}}$ \\
\hline 2145 & $2180-2120$ & $\mathrm{R}-\mathrm{N} \equiv \mathrm{C}$, isonitrile stretch $^{\mathrm{d}}$ \\
\hline 2110 & $2120-2100$ & aromatic $-\mathrm{N} \equiv \mathrm{C}$ stretch \\
\hline 1700 & $1730-1680$ & $\begin{array}{l}\mathrm{C}=\mathrm{O} \text { stretch } \\
\text { Also: carbonyl (aldehyde, ketone), carboxyl }\end{array}$ \\
\hline 1625 & $1650-1620$ & $\begin{array}{l}\mathrm{C}=\mathrm{N} \text { stretch } \\
\text { Also: } \mathrm{C}=\mathrm{O}, \mathrm{C}=\mathrm{C} \text { stretch }\end{array}$ \\
\hline 1560 & $1570-1540$ & $\begin{array}{l}\mathrm{C}=\mathrm{C} \text { stretch } \\
\text { Also: } \mathrm{C}=\mathrm{N} \text {, quinones, carboxylates, } \mathrm{NO}\end{array}$ \\
\hline 1460 & $1480-1440$ & $\mathrm{CH}_{2,3}, \mathrm{NH}_{2}, \mathrm{C}-\mathrm{OH}$ bending \\
\hline 1350 & $1370-1340$ & $\begin{array}{l}\mathrm{CH}_{2,3} \text { bending } \\
\text { Also: aromatic } \mathrm{C}-\mathrm{N} \text { bending, } \mathrm{N}-\mathrm{O} \text { stretch }\end{array}$ \\
\hline 1230 & $1250-1220$ & $\begin{array}{l}\mathrm{C}-\mathrm{O} \text { stretch } \\
\text { Also: } \mathrm{C}-\mathrm{OH} \text { stretch, ester }\end{array}$ \\
\hline
\end{tabular}

Notes.

a http://webbook.nist.gov/chemistry/

b https://webspectra.chem.ucla.edu/irtable.html

c Imanaka et al. (2004).

d Mutsukura (1999).

bands in the mid-infrared: (a) amines $\left(3800-2400 \mathrm{~cm}^{-1}\right)$, (b) nitriles (2300-2050 $\left.\mathrm{cm}^{-1}\right)$, and a (c) hetero-aromatics band $\left(1900-1000 \mathrm{~cm}^{-1}\right)$ (Mutsukura 1999; Imanaka et al. 2004). The assignment of these modes are provide in Table 3 . We have deconvolved the main mid-infrared spectral bands of all samples prepared with a $95 \% \mathrm{~N}_{2}$ concentration, corresponding to the following $\mathrm{N}_{2}: \mathrm{CO}_{2}: \mathrm{CH}_{4}$ mixtures: $95: 0: 5,95: 2.5: 2.5$, 
Amines

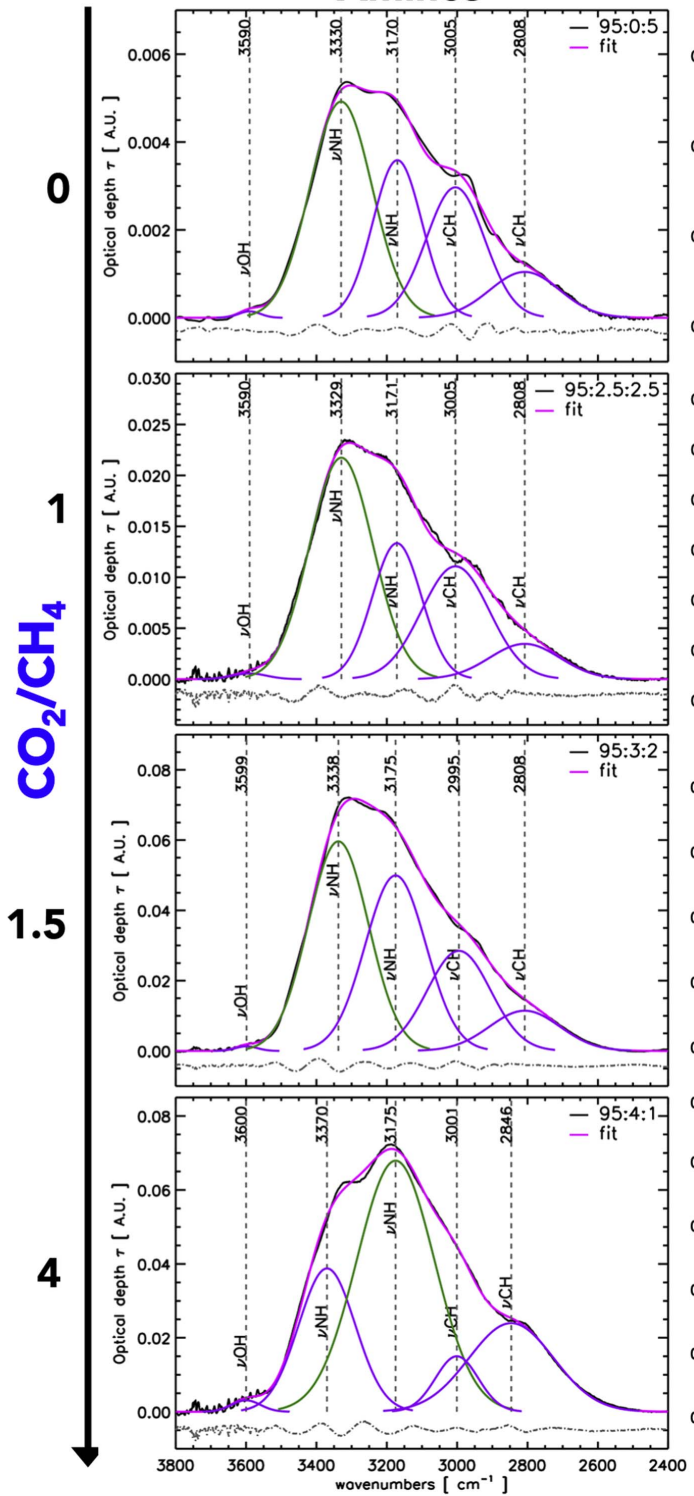

Nitriles
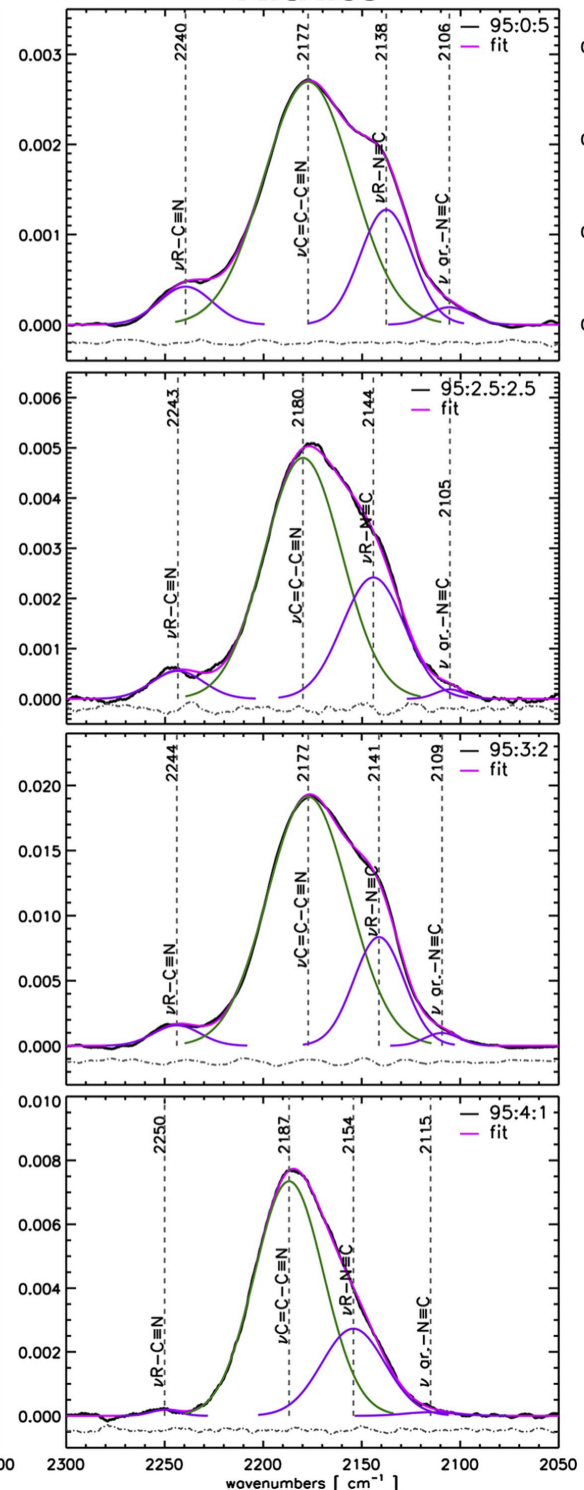

Hetero-aromatics
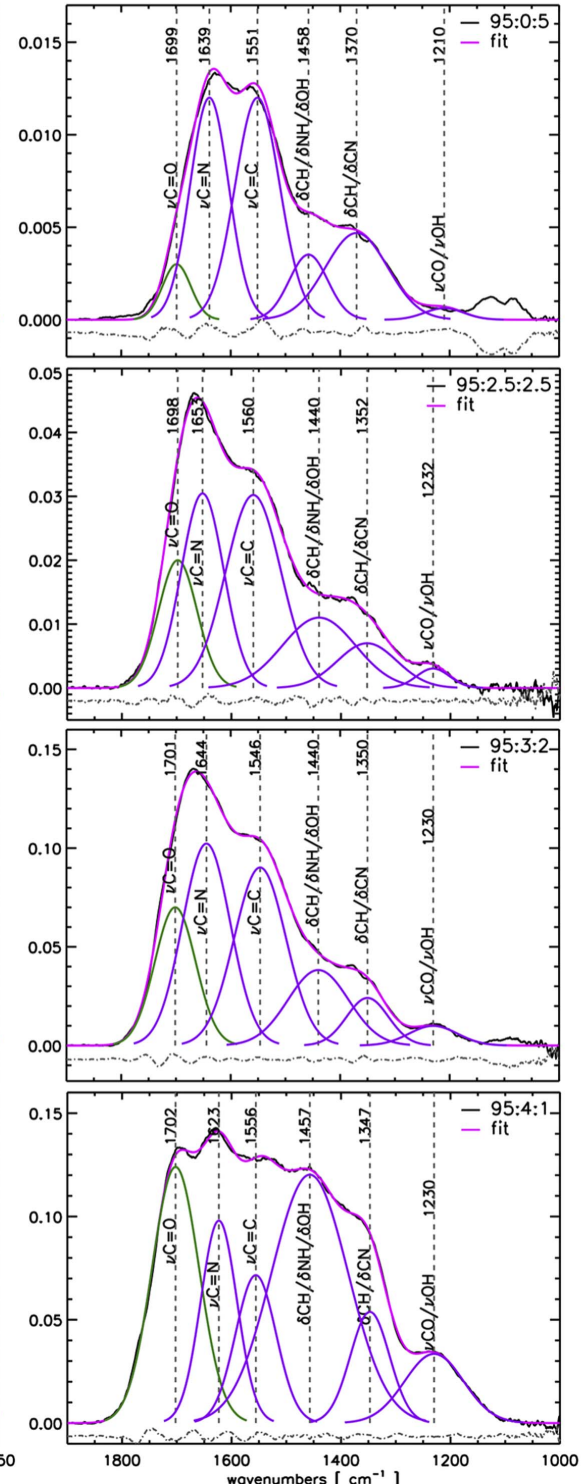

Figure 4. Spectral deconvolution of the main mid-infrared spectral bands (amines, nitriles, hetero-aromatics spanning $3800-1000 \mathrm{~cm}^{-1}$ ) of increasingly oxidized organic tholins. To illustrate the effect of the increasing $\mathrm{CO}_{2} / \mathrm{CH}_{4}$ ratio on the molecular structure, only tholins from the same series $\left(95 \% \mathrm{~N}_{2}\right)$ and varying $\mathrm{CO}_{2} / \mathrm{CH}_{4}$ ratios $=0,1,1.5$, and 4 , are used.

95:3:2, and 95:4:1, plotted in Figure 4. Such deconvolution provides further insight into the evolution of the molecular structure of organic aerosols as a function of their oxidation degree. In this first-order analysis, we use Gaussian functions to fit the most intense vibrational modes due to broad bonding distributions in the solid network that result in statistical bell curves, and neglect any perturbations to the line shape due to secondary resonances. Fitting vibrational bands is done by using the number of Gaussians needed to characterize the vibrational spectra of the $\mathrm{CO}_{2} / \mathrm{CH}_{4}=0$ tholin, which has the largest number of peaks. To fit the tholin spectra at different oxidation degrees, we have assumed that (1) the organic network of tholins may be similar to first order and (2) oxidation will produce new bonds that may shift the energies and abundances of functional groups.

For tholins prepared with increasing $\mathrm{CO}_{2} / \mathrm{CH}_{4}$ ratios, two main features appear in the amine band. These are attributed to the stretching modes of $\mathrm{NH}$ functions in amide groups at $\sim 3180 \mathrm{~cm}^{-1}$ and to aldehyde $\mathrm{CH}$ stretching modes at $\sim 2800 \mathrm{~cm}^{-1}$. In the nitrile band, increasing oxidation translates into a decrease of singly bonded and aromatic isonitriles at $\sim 2140 \mathrm{~cm}^{-1}$ and $\sim 2105 \mathrm{~cm}^{-1}$, respectively, and to the decrease of singly bonded nitrile modes at $\sim 2240 \mathrm{~cm}^{-1}$. In contrast at $\mathrm{CO}_{2} / \mathrm{CH}_{4}$ ratios greater than 1 , we note the effect of increasing oxidation on the heteroaromatic band: the unsaturated $\mathrm{C}=\mathrm{C}$ and $\mathrm{C}=\mathrm{N}$ modes decrease while the intensity of the carbonyl $(\mathrm{C}=\mathrm{O})$ band at $1700 \mathrm{~cm}^{-1}$ increases. This is confirmed by the formation of a new band in the region between 1550 and $1300 \mathrm{~cm}^{-1}$, attributed to bending modes in $\mathrm{NH}_{2}, \mathrm{CH}_{2,3}$, and $\mathrm{C}-\mathrm{OH}$ functional groups.

From the deconvolution of the amines band an increasing $\mathrm{CO}_{2} / \mathrm{CH}_{4}$ ratio results in the rapid evolution of the $\mathrm{NH}$ stretching mode associated with amides and to the aldehyde $\mathrm{CH}$ stretch. Although the primary $\mathrm{NH}$ bonds associated with amines are present and common for all samples, in samples with a $\mathrm{CO}_{2} / \mathrm{CH}_{4}=4$ ratio, the $\mathrm{NH}$ band associated with 


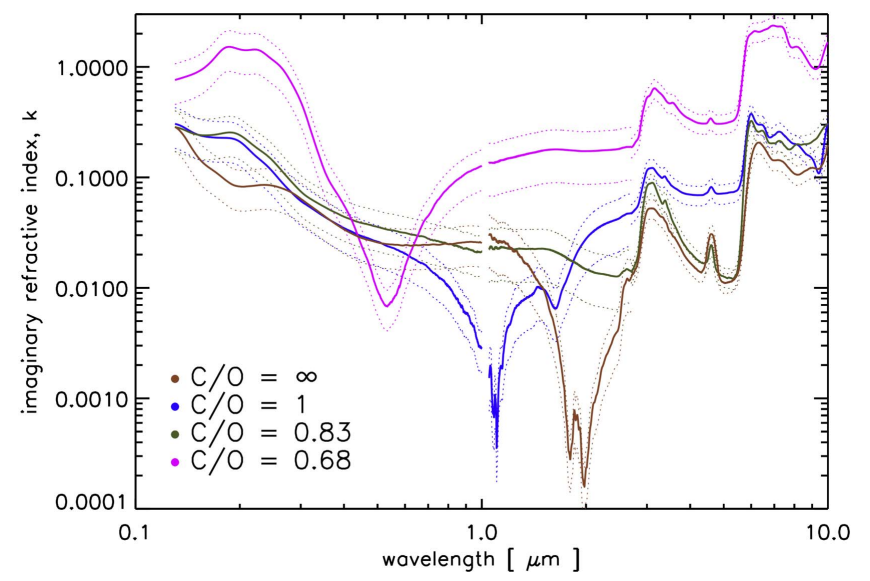

Figure 5. Imaginary refractive index $k$ spanning the VUV-Vis, NIR, and MIR ranges for tholins prepared at increasing $\mathrm{CO}_{2} / \mathrm{CH}_{4}$ or $\mathrm{C} / \mathrm{O}$ ratios. As oxidation increases, the absorption coefficient increases steeply in the ultraviolet range $(130-300 \mathrm{~nm})$, linearly in the mid-infrared, while the $k$ inversion region is blueshifted.

amides is relatively more intense. At maximum oxidation there is also a loss of the aliphatic $\mathrm{CH}$ stretching band, suggesting a decreased $\mathrm{C} / \mathrm{N}$ ratio in the most oxidized materials. The deconvolution of the nitrile band shows the progressive loss of isonitrile modes with increasing oxidation. Together with the loss of singly bonded nitriles, this suggests a gain in disorder in the structure of oxidized tholins. Mass loss through nitriles with respect to the amine and hetero-aromatic bands is a significant effect of oxidation.

Due to the large number of overlapping stretching and bending modes, deconvolution of the hetero-aromatic band is performed by simplifying the number of participant modes. The region between 1500 and $1300 \mathrm{~cm}^{-1}$ is attributed to a large peak, including the overlapping contribution of bending modes from $\mathrm{CH}, \mathrm{NH}$, and $\mathrm{OH}$ groups. As the $\mathrm{CO}_{2} / \mathrm{CH}_{4}$ ratio increases, the $\mathrm{C}=\mathrm{O}$ stretching mode at $\sim 1700 \mathrm{~cm}^{-1}$ increases, while the relative intensities of the $\mathrm{C}=\mathrm{N}$ and aromatic $\mathrm{C}=\mathrm{C}$ stretching modes decrease. In the plasma, oxidation is due to $\mathrm{O}$ atoms and $\mathrm{OH}$ radicals produced in the dissociation of $\mathrm{CO}_{2}$ and $\mathrm{CH}_{4}$ molecules. As the oxidation degree increases, the formation of $\mathrm{NH}$ and $\mathrm{C}-\mathrm{N}$ bonds are favored relative to $\mathrm{C}=\mathrm{N}$ bonds. We also note that, even without $\mathrm{CO}_{2}$ in the gas mixture, the $\mathrm{C}=\mathrm{O}$ mode is present in the reduced tholin. This could be due to surface oxidation by $\mathrm{O}_{2}$ in air while transporting the sample for analysis or to trace oxygen species present in the plasma.

\subsection{Absolute Imaginary Indices}

Transmission spectra in the VUV-visible, near-infrared, and mid-infrared ranges for each sample and the thicknesses listed in Table 1 are used to estimate the wavelength-dependent imaginary index $k(\nu)$, over this spectral region, by means of the relation,

$$
k(\nu)=\frac{\tau(\nu)}{4 \pi \nu d}
$$

where $\tau(\nu)$ is the optical depth, $\nu$ is the frequency, and $d$ is the sample thickness. The result is shown in Figure 5. The progressive increase of oxidation in these organic materials directly results in the increase of the absolute absorption over

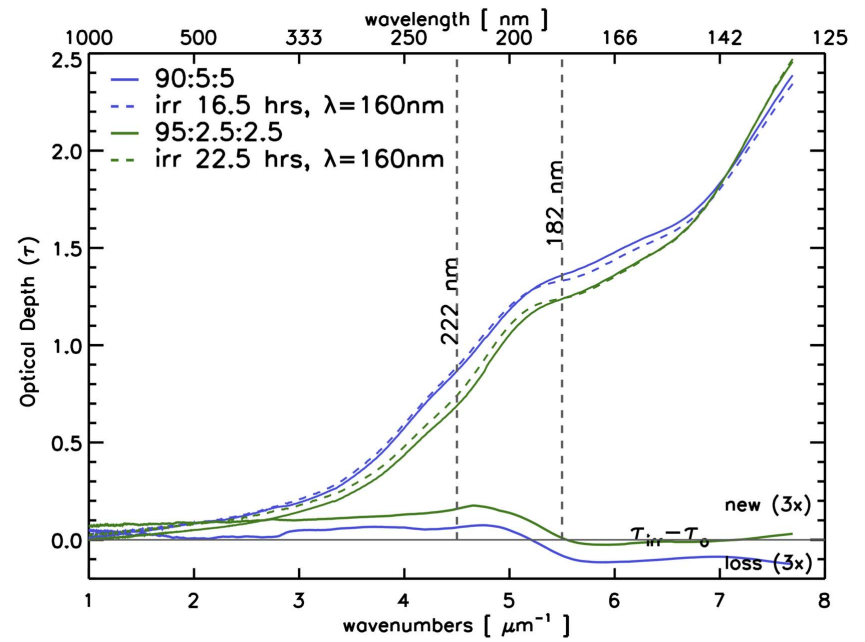

Figure 6. Vis-VUV spectra of oxidized tholins irradiated with VUV photons. The $\tau_{\text {irr }}-\tau_{o}$ lines around 0 show the new and lost band intensities (scaled by a factor of 3).

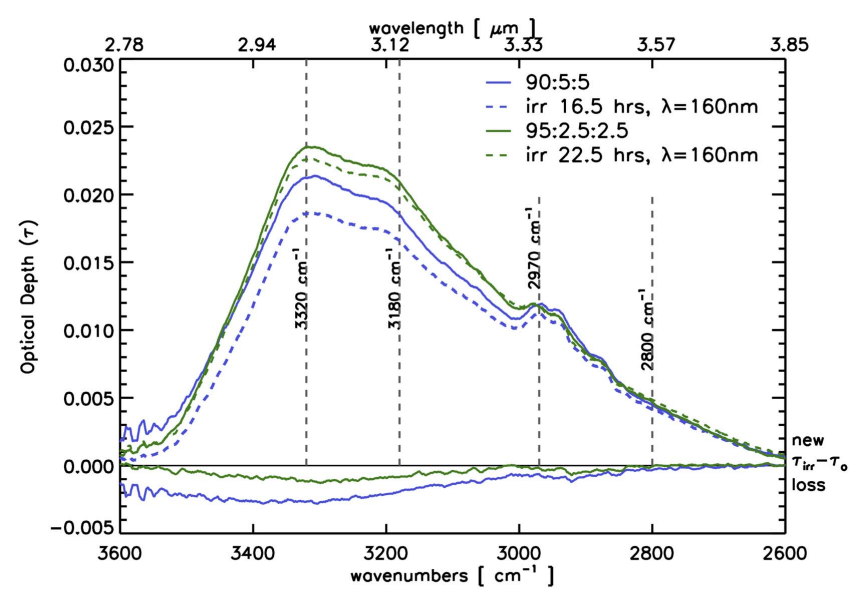

(a)

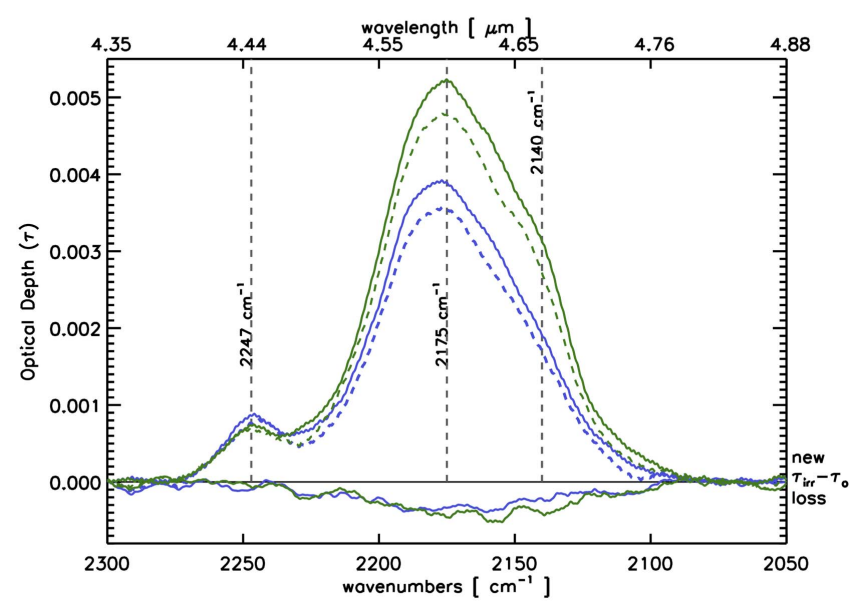

(b)

Figure 7. Mid-infrared spectra (normalized) of VUV-irradiated tholins showing the evolution of the (a) amine $\left(3600-2600 \mathrm{~cm}^{-1}\right.$ ) and (b) nitrile $\left(2300-2050 \mathrm{~cm}^{-1}\right)$ bands.

the entire wavelength range (from 0.13 to $9 \mu \mathrm{m}$ ). For the most oxidized tholin, $\mathrm{C} / \mathrm{O}=0.68$, the absolute absorption increases most prominently in the the VUV, $0.13-0.2 \mu \mathrm{m}$, and UV, 
$0.2-0.3 \mu \mathrm{m}$, in agreement with previous results reported in Gavilan et al. (2017).

In the mid-infrared the most absorptive region corresponds to the hetero-aromatic band, which increases linearly with the $\mathrm{C} / \mathrm{O}$ ratio. We also note a significant drop of $k$ over two orders of magnitude from the VUV to the visible region $(0.13-1 \mu \mathrm{m})$ for the most reduced sample, the Titan type tholin with $\mathrm{C} / \mathrm{O}=\infty$. McKay et al. (2001) showed that this drop is essential for reproducing the shape of Titan's geometric albedo.

The uncertainty in near-infrared absorption is larger due to the lower absorption indices in this region, where $k \leqslant 0.2$. The absorption in the near-infrared increases with oxidation due to the presence of second and third overtones of $\mathrm{NH}, \mathrm{NH}_{2}$, and $\mathrm{OH}$ vibrations. This region is also characterized by absorption minima. An inversion of $k$ is present at $1.8-2 \mu \mathrm{m}$ for the $\mathrm{C} / \mathrm{O}=\infty$ tholin, and the position of this inversion feature blueshifts toward $0.9-1.1 \mu \mathrm{m}$ for the tholin with $\mathrm{C} / \mathrm{O}=1$ and to $0.5-0.6 \mu \mathrm{m}$ for the tholin with $\mathrm{C} / \mathrm{O}=4$. However, this $k$ inversion is absent from the tholin corresponding to $\mathrm{C} / \mathrm{O}=0.83$ (90:6:4). Although this sample is more oxidized, as seen by the decrease in nitrile intensity and the oxidation of the amine and hetero-aromatic bands, the lack of a minimum imaginary absorption index is surprising. This may be explained by the peculiar plasma conditions produced at a ratio of $\mathrm{CO}_{2} / \mathrm{CH}_{4}=1.5$. At this ratio the plasma attains a peculiar (dis-)equilibrium where tholin production is enhanced rather than quenched. Further experiments with this particular gas mixture should unveil the gas kinetics of this plasma and its effects on aerosol production.

\section{VUV Irradiation of Tholins}

Two tholins prepared with a ratio of $\mathrm{CO}_{2} / \mathrm{CH}_{4}=1$ were irradiated with a monochromatic beam of $160 \mathrm{~nm}$ photons. The VUV-visible spectra of the samples were measured before, $\tau_{o}$, and after irradiation, $\tau_{\text {irr. }}$.

In all cases, a slight decrease in the spectral intensity in the $5.5-7 \mu \mathrm{m}^{-1}(200-150 \mathrm{~nm})$ region is attributed to the VUV photolysis of aromatic $\mathrm{C}=\mathrm{O}$ structures. In contrast, we note the intensity growth of the $3.5-4.5 \mu \mathrm{m}^{-1}(200-300 \mathrm{~nm})$ region, attributed to $\pi$-bond formation of the organic matrix. The photon flux that the beam line produces was measured using an AXUV100G photodiode. Using the known responsivity of this diode, at $\lambda=160 \mathrm{~nm}$ there are $6.7 \times 10^{11}$ photons $/ \mathrm{s} / 100 \mathrm{~mA}$. During the irradiation, the beam current was recorded every second and the total fluence was calculated based on the average beam current $(\sim 180 \mathrm{~mA})$. The 90:5:5 sample received a dose of $\sim 5 \times 10^{17} \mathrm{eV} \mathrm{cm}^{-2}$, while the 90:6:4 sample received a dose of $\sim 8 \times 10^{17} \mathrm{eV} \mathrm{cm}^{-2}$. The VUV spectra before and after irradiation are shown in Figure 6.

These results are consistent with previous VUV irradiation experiments performed by Gadallah et al. (2011) on hydrogenated amorphous carbon (HAC) materials. They used a deuterium lamp, whose emission peaks at $160 \mathrm{~nm}$, and irradiated carbonaceous thin films with doses of at least $10^{22}$ $\mathrm{eV} \mathrm{cm}^{-2}$. UV spectra of the irradiated HAC samples showed an increase of the $\mathrm{sp}^{2}$ carbon fraction, giving rise to $\pi-\pi^{*}$ electronic transitions peaking at $\lambda^{-1} \sim 4.6 \mu \mathrm{m}^{-1}(217 \mathrm{~nm})$. Our irradiation experiments confirm that structural modifications can arise at lower UV fluences in organic materials. During irradiation, the transmission of the $\mathrm{MgF}_{2}$ window could also evolve due to VUV photon absorption. However, as $\mathrm{MgF}_{2}$ is a nonorganic crystal, the growth of new bands in the $200-300 \mathrm{~nm}$ range is attributed to the evolution of the tholin.
The mid-infrared spectra of the VUV irradiated samples were measured before and after irradiation as shown in Figure 7. Following irradiation, the amine band shows the greatest loss in intensity. This loss is most marked for amines in the $3400-3200 \mathrm{~cm}^{-1}$ region, with a maximum loss at $\sim 3300 \mathrm{~cm}^{-1}$. The lower frequency modes in the amines region, including the $\mathrm{CH}$ and $\mathrm{OH}$ bands around $3000-2600 \mathrm{~cm}^{-1}$, are little affected. Following VUV irradiation, the nitrile band shows a general depletion with a maximum loss of conjugated nitriles. Both mass loss (desorption) and photolysis (inducing fragmentation and/or bond creation) could occur during the VUV irradiation of tholins. Further experiments are required to unveil which mechanism is responsible for the photo-degradation effects.

To compare the laboratory UV doses to the radiation from an exoplanet host star, we use the UV spectra of GJ876, an M dwarf observed by the International Ultraviolet Explorer (Linsky 2014). In this study, the spectral flux is rescaled to the distance of its habitable zone $(0.21 \mathrm{au})$. We consider the flux of GJ876 at $\lambda=160 \mathrm{~nm}$, of $\sim 8 \mathrm{erg} \mathrm{cm}^{-2} \mathrm{~s}^{-1}$, corresponding to the monochromatic photons used at the synchrotron. The laboratory doses are then equivalent to exposure times of $\sim 28 \mathrm{hr}$ (for the 90:5:5 tholin) and $\sim 45 \mathrm{hr}$ (for the 95:2.5:2.5 tholin) at 0.21 au of GJ876. Considering the low formation yields (and slow accumulation) of oxidized organic aerosols, it is likely that these will undergo photoprocessing during their lifetime in exoplanet atmospheres. The laboratory VUV-MIR spectra of photodegraded tholins could be relevant to future observations of exoplanetary hazes.

\section{Discussion}

Future space telescopes like ARIEL $^{9}$ and LUVOIR $^{10}$ will enable the investigation of exoplanets ranging from Jupiterand Neptune-size down to super-Earth and Earth-size. ARIEL instruments are expected to cover the visible and infrared 0.5-7.8 $\mu \mathrm{m}$ range (Tinetti et al. 2016), while LUVOIR will enable multiwavelength observations covering the ultraviolet to near-infrared from 0.2 to $2.5 \mu \mathrm{m}$.

Our spectroscopic VUV-MIR study covering the $0.13-10 \mu \mathrm{m}$ range can thus provide relevant laboratory constraints for the future observations of exoplanet atmospheres. The spectra of oxidized aerosols signals a distinct large absorption in the $0.13-0.4 \mu \mathrm{m}$ range and a flatter but strong absorption band in the 6-7.5 $\mu \mathrm{m}$ range relative to reduced aerosols. This high absorption coefficient may increase the detectability of exoplanet atmospheres where hazes form under oxidizing conditions. Together with a weaker nitrile band and a minimum absorption window in the visible range, these constitute potential observables of atmospheric oxidation. Organic aerosols constitute potential exoplanet atmospheric biosignatures, as organic hazes can be derived from biogenic methane (Catling et al. 2018), although abiotic sources are not discarded. While the simultaneous detection of $\mathrm{CO}_{2}$ and $\mathrm{CH}_{4}$ in a habitable exoplanet atmosphere has been recently proposed as a potential biosignature (Krissansen-Totton et al. 2018), ensuing oxidized organic hazes could also constitute observable biosignatures.

\footnotetext{
ARIEL: Atmospheric Remote-Sensing Infrared Exoplanet Large-survey.

${ }^{10}$ LUVOIR: Large UV/Optical/IR Surveyor.
} 


\section{Conclusions}

We have produced laboratory analogs to oxidized organic aerosols. These were prepared in increasingly oxidizing plasmas from mixtures of $\mathrm{N}_{2}: \mathrm{CO}_{2}: \mathrm{CH}_{4}$, characterized by an increasing $\mathrm{CO}_{2} / \mathrm{CH}_{4}$ ratio from 0 (fully reducing) to 4 . These correspond to atmospheres with $\mathrm{C} / \mathrm{O}$ ratios from $0.68,0.83$, 1 , and $\infty$, i.e., representative of carbon-poor, solar-like to carbon-rich atmospheres. The samples were characterized via transmission spectroscopy from the vacuum-ultraviolet to the mid-infrared.

1. The molecular and electronic structure of these materials is dominated by the $\mathrm{CO}_{2} / \mathrm{CH}_{4}$ ratio in the $\mathrm{N}_{2}: \mathrm{CO}_{2}: \mathrm{CH}_{4}$ gas mixture. Ultraviolet spectroscopy revealed the presence of $\pi-\pi^{*}$ and $n-\pi^{*}$ transitions in the $2<\lambda^{-1}<$ $5 \mu \mathrm{m}^{-1}(200-500 \mathrm{~nm})$ range in all samples. These are attributed to electronic transitions in amine bonds for tholins prepared in fully reducing conditions. As the $\mathrm{CO}_{2} / \mathrm{CH}_{4}$ ratio increases new bands emerge in this range, attributed to electronic transitions in hydroxyl and carboxyl bonds. For all cases, a large absorption band in the far-ultraviolet is present and is attributed to $\sigma-\sigma^{*}$ and $n-\sigma^{*}$ electronic transitions.

2. Infrared spectroscopy revealed the formation of oxygenated bonds with an increasing $\mathrm{CO}_{2} / \mathrm{CH}_{4}$ ratio including stretching modes from $\mathrm{NH}, \mathrm{OH}, \mathrm{C}=\mathrm{O}$, and $\mathrm{C}=\mathrm{N}$ and the formation of a new band in the $\sim 1400-1300 \mathrm{~cm}^{-1}$ region, which could be attributed to $\mathrm{NH}, \mathrm{C}-\mathrm{N}, \mathrm{OH}$, and $\mathrm{C}-\mathrm{O}$ bonds, as expected in $\mathrm{CHON}$-rich organic matter. The decomposition of the nitriles band revealed the loss of aromatic nitriles and singly bonded $\mathrm{R}-\mathrm{N}=\mathrm{C}=\mathrm{N}$ modes with oxidation.

3. The absolute absorption index increases linearly for all samples with increasing degrees of oxidation. The absorption is greatest in the VUV-UV range from 0.13 to $3 \mu \mathrm{m}$ for the most oxidized samples, which are also the most opaque in the $6-10 \mu \mathrm{m}$ hetero-aromatic band region. Oxidized aerosols show a weaker nitrile band and a blueshifted absorption minimum compared to reduced aerosols.

4. VUV irradiation of oxygenated aerosols revealed the formation of new $\pi-\pi^{*}$ bands in the $4<\lambda^{-1}<5 \mu \mathrm{m}^{-1}$ $(200-250 \mathrm{~nm})$ region and the loss of electronic bands below $200 \mathrm{~nm}$, corresponding to the formation of $\mathrm{NH}$, $\mathrm{C}-\mathrm{N}$, and $\mathrm{OH}$ bonds and the loss of amines and isonitriles observed in their MIR spectra.

5. Our results suggest that oxidative plasma chemistry can result in solids with complex oxygenated bonds leading to solids that are more opaque in the VUV-MIR range. Because of their high absorption properties, oxidized organic aerosols could improve the detectability of exoplanet atmospheres. This spectral signature is indicative of the presence of oxic atmospheric conditions and could offer a potential biosignature for photosynthetic life.

We thank the staff at the ASTRID2 synchrotron for their help and access to beam time. We thank A. Giuliani for the preliminary VUV measurement of the reduced tholin sample at the DISCO beam line of the SOLEIL synchrotron (project No. 20160171) and E. Dartois for kindly supplying the DISCOadapted sample holder. We thank A. Maturilli for his help with the near-infrared measurements at the Planetary Emissivity Laboratory (DLR). L.G. recognizes support for this visit from the Europlanet Research Infrastructure funded by the European
Union's Horizon 2020 research and innovation programme under grant agreement No. 654208. N.J.M. recognizes support from the COST Action TD1308 Origins. N.C. and L.G. thank the European Research Council for funding via the ERC PrimChem project (grant agreement No. 636829.).

Software: IDL MPFIT (Markwardt 2009).

\section{ORCID iDs}

Lisseth Gavilan (ํ) https://orcid.org/0000-0001-8645-8415 Nathalie Carrasco iㅣ https://orcid.org/0000-0002-0596-6336

\section{References}

Ari, T., \& Güven, M. H. 2000, JESRP, 106, 29

Arney, G. N., Meadows, V. S., Domagal-Goldman, S. D., et al. 2017, ApJ, 836,49

Barnes, E. E., \& Simpson, W. T. 1963, JChPh, 39, 670

Catling, D. C., Krissansen-Totton, J., Kiang, N. Y., et al. 2018, AsBio, 18, 709

Duarte, A. A., Gomes, P. J., Ribeiro, J. H. F., et al. 2013, EPJE, 36, 98

Feulner, G. 2012, RvGeo, 50, RG2006

Fleury, B., Carrasco, N., Millan, M., Vettier, L., \& Szopa, C. 2017, E\&PSL, 479,34

Gadallah, K. A. K., Mutschke, H., \& Jäger, C. 2011, A\&A, 528, A56

Gavilan, L., Alata, I., Le, K. C., et al. 2016, A\&A, 586, A106

Gavilan, L., Broch, L., Carrasco, N., Fleury, B., \& Vettier, L. 2017, ApJL, 848, L5

Haqq-Misra, J. D., Domagal-Goldman, S. D., Kasting, P. J., \& Kasting, J. F. 2008, AsBio, 8, 1127

Harshbarger, W. R. 1971, JChPh, 54, 2504

Hornback, J. 2005, Organic Chemistry (2nd ed.; Boston, MA: Cengage Learning)

Hubin-Franskin, M.-J., Delwiche, J., Giuliani, A., et al. 2002, JChPh, 116, 9261

Imanaka, H., Khare, B. N., Elsila, J. E., et al. 2004, Icar, 168, 344

Jacobson, M. C., Hansson, H. C., Noone, K. J., \& Charlson, R. J. 2000, RvGeo, 38, 267

Jones, A. P. 2012, A\&A, 540, A2

Kasting, J. F. 1993, Sci, 259, 920

Krissansen-Totton, J., Olson, S., \& Catling, D. C. 2018, SciA, 4, eaao5747

Lavvas, P., \& Koskinen, T. 2017, ApJ, in press (arXiv:1708.09257)

Lavvas, P., Yelle, R. V., Koskinen, T., et al. 2013, PNAS, 110, 2729

Linsky, J. 2014, Chall, 5, 351

Madhusudhan, N., Agúndez, M., Moses, J. I., \& Hu, Y. 2016, SSRv, 205, 285

Mahjoub, A., Carrasco, N., Dahoo, P.-R., et al. 2012, Icar, 221, 670

Markwardt, C. B. 2009, adass XVIII, 411, 251

Marley, M. S., Ackerman, A. S., Cuzzi, J. N., \& Kitzmann, D. 2013, in Clouds and Hazes in Exoplanet Atmospheres, ed. S. J. Mackwell et al. (Tucson, AZ: Univ. Arizona Press), 367

McKay, C. P., Coustenis, A., Samuelson, R. E., et al. 2001, P\&SS, 49, 79

Mutsukura, N. 1999, TSF, 349, 115

Palmer, M. H., Ridley, T., Hoffmann, S. V., et al. 2015, JChPh, 142, 134302

Pavlov, A. A., Brown, L. L., \& Kasting, J. F. 2001, JGR, 106, 23267

Robertson, J., \& O’Reilly, E. P. 1987, PhRvB, 35, 2946

Sciamma-O'Brien, E., Dahoo, P.-R., Hadamcik, E., et al. 2012, Icar, 218, 356

Swain, M. R., Tinetti, G., Vasisht, G., et al. 2009a, ApJ, 704, 1616

Swain, M. R., Vasisht, G., \& Tinetti, G. 2008, Natur, 452, 329

Swain, M. R., Vasisht, G., Tinetti, G., et al. 2009b, ApJL, 690, L114

Szopa, C., Cernogora, G., Boufendi, L., Correia, J. J., \& Coll, P. 2006, P\&SS, 54,394

Tannenbaum, E., Coffin, E. M., \& Harrison, A. J. 1953, JChPh, 21, 311

Tauc, J., Grigorovici, R., \& Vancu, A. 1966, PSSBR, 15, 627

Tinetti, G., Drossart, P., Eccleston, P., et al. 2016, Proc. SPIE, 9904, 99041X

Trainer, M. G., Pavlov, A. A., Dewitt, H. L., et al. 2006, PNAS, 103, 18035

Urbach, F. 1953, PhRv, 92, 1324

Venot, O., Bénilan, Y., Fray, N., et al. 2018, A\&A, 609, A34

Venot, O., Hébrard, E., Agúndez, M., Decin, L., \& Bounaceur, R. 2015, A\&A, 577, A33

von Paris, P., Rauer, H., Lee Grenfell, J., et al. 2008, P\&SS, 56, 1244

Wilson, E. H., \& Atreya, S. K. 2004, JGRE, 109, E06002

Wolf, E. T., \& Toon, O. B. 2010, Sci, 328, 1266

Workman, J. 2016, Concise Handbook Of Analytical Spectroscopy, The: Theory, Applications, And Reference Materials (Singapore: World Scientific)

Xu, K., Amaral, G., \& Zhang, J. 1999, JChPh, 111, 6271 